\title{
Commentary
}

\section{Proposed aims, organization, and activities of a division of clinical pharmacology}

\begin{abstract}
The general aim of a division of clinical pharmacology is to provide opportunity for the work of present clinical pharmacologists, to encourage the development of improved methods of research, and, in particular, to foster the training of young men in this discipline. Specific areas of research, teaching, and service can already be foreseen. The best administrative home of the division will probably be a department of pharmacology, although special arrangements regarding budget, personnel, and appointments will probably be necessary. Training in clinical pharmacology should be from 1 to 3 years. The trainee should have already received a background of clinical experience, and this may be further strengthened during his specific training in clinical pharmacology. Courses in advanced pharmacology and statistics are obviously necessary. Each division will probably find it necessary to create at least one new course dealing with the special problems of human pharmacology, discussed preferably in an informal seminar environment.
\end{abstract}

Edward A. Carr, Jr., M.D. Ann Arbor, Mich.

Departments of Internal Medicine and Pharmacology (Program in Investigative Clinical Pharmacology), University of Michigan

Approximately twenty medical schools in the United States have already established or made specific plans to establish divisions, units, or programs in clinical pharmacology. Some of these have developed over a period of a decade or more, roughly in parallel with the development of individual clinical pharmacologists in these schools. Other divisions of clinical pharmacology have been recently created, under circumstances of somewhat forced

Received for publication June 26, 1963. development, to meet the rapidly expanding needs. The purpose of the present communication is to suggest that the experience of the clinical pharmacology groups that have developed over the past decade is relevant to the further development of the field, and should be applied to promote reasonably rapid but orderly expansion in this area. Since I do not believe that advice limited to vague generalities will be of much help to those responsible for the development of new divisions, an outline of specific proposals is presented. 


\section{Background}

At least seven factors have provided the impetus for the development of clinical pharmacology.

A. With the explosive increase in the number of new compounds for clinical testing after World War II, differences between human reactions to drugs and the reactions of other species became increasingly apparent and disturbing. Comparative pharmacology ceased to be a curiosity and became, literally, a life-and-death matter.

B. This same explosive development led to a parallel explosive development of dubious therapeutic claims.

C. A frustrating sense of wasted opportunities began to affect all those who observed the increasing divergence between the basic sciences and clinical medicine. Medicine began to develop its own "two cultures" problem.

D. A few techniques of special applicability to man, e.g., the double-blind technique of drug testing, were developed.

E. In certain new fields, e.g., the clinical use of radioisotopes, the demand for human use grew quickly. This created the need for more background work in experimental pharmacology. In these rapidly expanding areas of human use, clinical medicine threatened to outrun its supply lines.

F. A great deal of the work of instruction in therapeutics fell to the detail men of the pharmaceutical industry, by default.

G. The thalidomide tragedy brought the government much more energetically into the field.

\section{The present state of the field}

A. No definition of clinical pharmacology, satisfactory to everyone, has been developed. However, the term "clinical pharmacology" will be used here, as it has taken precedence over certain competing terms, e.g., human pharmacology, even though some of us might prefer other terms.

B. At various organizational meetings devoted to clinical pharmacology, the vari- ation in background of the individuals attending the meetings has been notable. Two features do characterize clinical pharmacologists: experience in carrying out investigations in man, and a desire to apply the principles of basic sciences, especially pharmacology, to such investigations. As will be discussed below, these two necessary conditions should not necessarily be sufficient conditions to characterize a clinical pharmacologist.

C. The most notable present trends in the programs already developed are the availability of fairly large sums of money to support training programs, the general agreement that any training program should include a course in statistics and advanced courses in pharmacology, and the realization that clinical pharmacologists will have to supply many services in connection with the new regulations of the Food and Drug Administration. Two other trends are disturbing. Certain rump groups have moved to set up premature organizations to act as "certifying boards" in clinical pharmacology. A few individuals may have obtained training grants in support of clinical pharmacology, and then devoted these grants to standard clinical investigation in a given subspecialty without development of a broad clinical pharmacology program. These two trends must be curbed.

\section{Aims of a division of clinical pharmacology}

A. General aims. Regardless of minor quarrels in definition, most educated persons would agree that certain activities represent "investigations in the basic sciences" and certain other activities represent "the practice of medicine." It is now recognized that the transfer of information from the first to the second usually involves two intermediate stages: a stage of animal experimentation consciously planned as a preliminary to investigation in man, and a phase of investigation in man before general use in practice. Whenever human investigation goes beyond the making of sim- 
ple recordings * from untreated patients and involves administration of some substance to man, special problems arise. These include problems of ethics, safety, evaluation, and required pay-off (i.e., one may demand that a study involving a risk to humans have a higher probability of yielding valuable information than a study carried out in vitro). The crucial characteristic of clinical pharmacology is that it differs from pure investigation in the basic sciences in that the investigator may have a practical (even if far distant) end in mind; simultaneously, the clinical pharmacologist differs from the pure practitioner of medicine in that the former is trying to find out "how things work" in man, while the latter is trying to make the most efficient use of the information that is already established. The clinical pharmacologist differs from certain other clinical investigators in that the former not only recognizes the special problems involved in administering substances to humans for experimental purposes but also wishes to establish as many general principles as possible in this wide field. Moreover, he wishes to replicate himself by training others in this general field, rather than simply training others in the clinical subspecialty of his own particular competence.

Certain distinctions are not germane to clinical pharmacology. For example, it does not matter whether one is studying a new compound or trying to find out more about an old one, whether it is to be used for diagnostic or for therapeutic purposes, whether the substance acts by combining with enzymes, emitting beta particles or other mechanism, or whether undesirable effects are mediated by antibodies, crystals in the urine, or other means.

Perhaps the best analogy is this: between the "chromosomal" thinking of the basic scientist and the "ribosomal" activi-

"The term "simple recordings" is intended to convey the idea that one has not deliberately altered the system by administration of a drug. The term is not intended to disparage the elegance and importance of much research by physiologists. ties of the clinician, messenger RNA is needed. Clinical pharmacology aspires to be the messenger RNA.

B. Specific aims. A division of clinical pharmacology should be active in teaching, research, and service. Only a brief outline of the major aims of each is presented in this section on aims; further details will be found in section $V$.

In research, three areas deserve major attention: first, the problem of dangerous and (at present) unpredictable adverse reactions to new drugs in man, such as bone marrow depression, anaphylaxis, liver damage, etc.; second, the development of therapeutics as a more rational branch of medicine than it is now; and third, the exploitation of newer areas developed by basic scientists. Recent major advances in clinical medicine have continued to exploit, for example, basic discoveries made in immunology during the last century and principles of chemotherapy developed early in this century. This "fossil fuel" of older discoveries is being used up rapidly, and we must look into newer discoveries of the basic scientists for sources for further improvement in medicine.

In teaching, the chief functions should be two: to train other investigators in clinical pharmacology, and to provide an approach to more rational therapeutics.

In service, the chief problem is different from that in teaching and research. It will be all too easy for clinical pharmacologists to find areas in which they can render service (see below). But clinical pharmacologists must carefully avoid creating artificial needs for themselves. Thus, a clinical pharmacologist must assist investigators through a maze of F. D. A. regulations but he should be careful not to promote additional regulations and further bureaucracy, simply to create an additional demand for his own services.

\section{Organization of a division of elinical pharmacology}

A. Administrative home. Among the basic sciences there can be no doubt that 
pharmacology must be the basis of clinical pharmacology. Among clinical departments the situation cannot be described so dogmatically. Throughout this discussion the department of internal medicine will be used as the reference clinical department, as it represents, in many schools, the clinical department most likely to participate in clinical pharmacology. But other clinical departments, e.g., anesthesiology, may supply major clinical strength to the division. In any event, a logical arrangement would place the division of clinical pharmacology in the department of pharmacology, with the majority of the members of the division also holding appointments in clinical departments such as internal medicine. Certain individuals from other disciplines, e.g., physiology, may well hold positions in a division of clinical pharmacology. Although such other disciplines would not have major representation, numerically, in a division of clinical pharmacology, those individuals from other disciplines who do become members of a division of clinical pharmacology should receive treatment that is scrupulously equal to that given to persons connected with the major department of the division. In many, probably most, medical schools the best interests of all departments concerned will be served by such an arrangement, which recognizes, realistically, the importance of the pharmacologic basis of clinical pharmacology, the likelihood that several clinical departments may form useful associations with it, and the fact that activities in the department of internal medicine are often more applicable to clinical pharmacology than to activities in certain other clinical departments.

B. Budget. Although a separate division rather than a separate department, clinical pharmacology should be supported by its own training grant or in some other way have its budget allotted separately. This will prevent the problem that has been reported to plague other types of scientific programs in some schools: Department A is already paying large salaries to highappointment loafers who spend most of their time in extraneous activities, furnishing little service to the department. This means that there is little money available for those who are to do the work of the department. Meanwhile, Department B already has a bad tradition of inequality in its service demands, and tends to seize younger members to do all the work. Department B, therefore, would ordinarily be inclined to seize the younger members of the clinical pharmacology division and make them slave very hard for their wages. The best solution is to assign clinical pharmacology its own tasks and its own funds. Then, for example, a clinical pharmacologist who is appointed assistant professor in internal medicine is not using up funds that the professor of medicine should be giving to full-time internists; the same applies to appointments in the pharmacology department. (The matter of service requirements is further discussed below.)

C. Personnel. The director and most of the permanent staff of a clinical pharmacology division should each meet four criteria. First, they should have had training and experience in pharmacology at an advanced level, beyond that of the usual course given to medical students, though this does not imply the absolute necessity of an advanced degree in pharmacology. Second, they should have sufficient clinical experience and competence to be accepted and trusted by their clinical colleagues in some area of clinical medicine, though this does not imply the absolute necessity of board certification. Third, they should have had thorough training in clinical pharmacology as such. At present, there is only one such group, the "first generation" of clinical pharmacologists, consisting of individuals who have had formal training in pharmacology and clinical medicine, and have also spent several years carrying out a program of self-training in clinical pharmacology. It appears unwise to turn the direction of a division of clinical pharmacology over to an individual who has not had considerable experience and training in clinical pharmacology. Therefore, the 
"first generation" has the duty to organize and direct divisions of clinical pharmacology. But the "first generation" of clinical pharmacologists is obligated to devote prompt attention to training a "second generation," who will in later years assume the direction of such divisions. Thus, the fourth requirement becomes this: the permanent staff of a clinical pharmacology division should be willing to devote themselves to the teaching and development of this area as a field of interest in its own right.

It is obvious that each division will be largely molded by the personal stamp of its own staff. This is as it should be in such a young field. There is every reason to foster such individualism, provided no attempt is made to set the pattern of clinical pharmacology in a rigid, premature mold. After twenty-five years, the "second generation" of clinical pharmacologists may wish to get together and structure the field more rigidly, but this is a matter for future decision.

D. Appointments. The director of a division of clinical pharmacology should ordinarily hold an appointment in a clinical department (usually internal medicine) equal to that of the head of another division, such as hematology, infectious disease, etc. His appointment in the department of pharmacology should be equal to that given to the senior members of the department next below the chairman. Other permanent members of the staff should have tenure appointments in pharmacology and internal medicine (or, in special instances, other clinical departments). Thus, permanent appointment in the division of clinical pharmacology would usually be a permanent appointment in both pharmacology and internal medicine, as individuals holding permanent appointments in a division of clinical pharmacology should be genuinely qualified to hold such ranks. One exception is foreseen. Under certain circumstances an individual with special skills, e.g., a statistician, may hold a major appointment in clinical pharmacology without a clinical appointment.
Since the entire concept of the field is centered about its bridging function, individuals not qualified in both areas would usually not hold permanent appointments in the division, with the exception of special resource persons just mentioned.

Members of the staff who have not reached permanent status should hold an appointment such as assistant professor, instructor, etc., in one department, either pharmacology or internal medicine. If qualified to hold a similar appointment in the other department, they may do so. Otherwise, they may hold an appointment such as teaching fellow, research fellow, or clinical fellow in the other department. Thus, one individual may be an assistant professor in pharmacology and assistant professor in internal medicine, while another with less clinical experience may be an assistant professor in pharmacology and clinical fellow in internal medicine. A third individual may be an assistant professor in internal medicine and research fellow in pharmacology. In this way no one would be asked to serve in a department in which he had no appointment, yet individuals would not be given appointments beyond their qualifications simply as "prestige" appointments.

Ordinarily, the pay and perquisites of each member of the division would be based upon his highest appointment.

E. Trainees. Trainees should be in one of the following catagories: (1) candidates for the permanent staff of the division of clinical pharmacology, (2) candidates for permanent staff positions in divisions of clinical pharmacology elsewhere, or (3) candidates for any regular position in a clinical department or pharmacology department in which the individual, even if no longer acting as a full-time clinical pharmacologist, can use his special training in clinical pharmacology to advantage. It is also recognized that (4) the practice of medicine in the community might well be improved if individuals who had special training in clinical pharmacology would enter it. Last, it is expected that (5) some 
trainees will subsequently find positions in governmental or industrial clinical pharmacology.

Training in clinical pharmacology should be from 1 to 3 years. As present trends in medical education already use up many precious years of a young man's time, we must weigh very carefully each additional year we add. It is presumed that the trainee in clinical pharmacology will already have had part of his training in clinical medicine and in pharmacology; therefore, he should be able to apply this training in clinical medicine and in pharmacology toward clinical pharmacology, and thus avoid the necessity of piling another full program of training on one he has already received. Though a 3 year program may be desirable in certain instances, the training program in a division of clinical pharmacology should more commonly be 2 years. Upon entry, the trainee should have already had an internship, some residency training or an internship, and some advanced pharmacology training. During his 2 years of specific training in clinical pharmacology, he should attempt to develop at least one clinical skill, take additional basic pharmacology courses, participate in a regular seminar in human pharmacology, take a course in statistics, obtain practical experience in teaching therapeutics, carry out a research project in human pharmacology together with supporting animal work, and obtain practical service experience in one or more of the areas listed under V, C.

The 1 year program would ordinarily be given to individuals with special needs, e.g., those described under IV, E. 3 and IV, E. 4 (above). Thus, a person with a Ph.D. degree in pharmacology, planning a fulltime career in basic pharmacology, may elect to take 1 year in clinical pharmacology to strengthen his own knowledge of human pharmacology; during this year, he may well be able to assist in a clinical study carried out, for example, by the department of anesthesiology. As another example, a cardiovascular clinical group may be grooming one of its residents for a permanent position with them. They may wish to have him spend a year of training in clinical pharmacology and then supply them with this special skill as a member of their group.

F. Space. The division should have its offices in close juxtaposition to the department of internal medicine or the department of pharmacology. It should also have laboratories for supporting animal work, and space for clinical investigation. Thus, the division should have laboratory space in connection with internal medicine, laboratory space in connection with pharmacology, and offices adjacent to one of these sets of laboratories. The necessity for travel from one area to another may impose loss of time on the members of the division but this is a necessary price to pay. Ambassadors who object to travel have chosen the wrong career!

\section{Functions of the division (please note III, B, 1)}

\section{A. Teaching.}

1. The division should organize the training of clinical pharmacologists. It should be directly responsible for conducting a regular seminar in human pharmacology.

2. The division should be in charge of teaching rational therapeutics based upon pharmacology to third and fourth year medical students, to the house staff, and to postgraduate groups. (See also V, C, 1, next page.)

3. Whenever possible, each member of the division should also give certain lectures in the basic second year pharmacology course, if for no other reason than for the good of his own soul.

4. Divisions of pharmacology should probably avoid heavy participation in mammoth undertakings to integrate all the basic sciences and all the clinical specialties into one huge correlation program. Integration among several basic sciences has proved successful in some schools, and integration among several clinical specialties 
may be desirable and possible. But the difficulties of integration between basic science and clinical medicine are now such that clinical pharmacology, in attempting to solve this problem, should at present limit its objectives to the areas discussed above.

\section{B. Research.}

1. Members of the division, particularly in the early part of their careers, may carry out any bona fide research project in basic science to master principles and techniques.

2. Similarly, clinical pharmacology of the routine therapeutic trial type may also be carried out as a necessary research function of the department.

3. However, the principal research desired is the careful observation of the effect of drugs in selected patients in order to learn more about human pharmacology, and the imaginative development of animal or other basic techniques to improve the predictability of undesirable reactions in man.

4. On many occasions it may be desirable for a member of the division to do detached service with a well-established clinical investigator. There is no objection to this, provided, however, the detachment is not too complete. Thus, a laboratory meeting of the division should be held once a week and all members carrying out research in the division should take part. At this time, each trainee will learn about the problems of clinical pharmacology in clinical areas other than the one in which he is interested, and will also submit his own work to the criticism of his colleagues. Experience suggests that members of a division must avoid being seduced by the advantages of animal work to the point that they abandon the more difficult investigations in man.

5. The conduct of routine, unimaginative drug testing has not been mentioned in the description of research activities thus far. This omission is anything but accidental. Any division of clinical pharmacology that allows itself to be converted into a mill grinding out a stream of drug tests will certainly have no time to contribute to a solution of the many fascinating problems that should concern the true clinical pharmacologist, i.e., the absorption, distribution, elimination, special toxicity, usual and unusual effects, etc., of drugs in man. Therefore, the degeneration of clinical pharmacology into simple drug testing would be a development as unfortunate as it is unnecessary.

\section{Services.}

1. Each individual in the division, insofar as his clinical competence permits, should render clinical service such as ward rounds, outpatient service, etc.

2. The development of a special therapy clinic to carry out certain projects in experimental therapeutics should be considered. The specific type of work to be carried out in the special therapy clinic may vary from year to year, and the details of admission of a patient to the special therapy clinic require careful consideration from the ethical, clinical, financial, and legal standpoints. The experience of clinical research units to date suggests that such special arrangements are indeed feasible and successful.

3. The clinical pharmacologist must be fully cognizant of the details and implications of F. D. A. regulations, the practical methods of meeting requirements of the law, etc. He must give practical aid to other investigators and to the school itself in these matters.

4. Clinical pharmacologists should be available to give advice in the conduct of research in man to any other group that asks for such advice, within the school.

5. Clinical pharmacologists should play a major role in the organization and function of the poison control center of the medical school's hospital.

6. Clinical pharmacologists should be available for clinical consultation in matters of undesirable reactions to drugs, etc. They should organize and direct a program for collection and reporting of adverse reactions to drugs within a medical center. 
7. The Pharmacy and Therapy (or Formulary) Committee should have the services of a clinical pharmacologist.

8. Each school, to make best use of a division of clinical pharmacology, should also consider its own peculiar characteristics as a medical school, and determine the ways in which a division of clinical pharmacology can best be fitted to these peculiar characteristics by carrying out additional special duties.

9. A serious warning should be mentioned here. Unless the duties of each member of the division are carefully spelled out, he is likely to be either hopelessly overworked or underutilized. The principle of " $1 / 3-1 / 3-1 / 3$ " should be considered here. Ordinarily, a clinical pharmacologist holding an appointment in pharmacology and in a clinical department would owe the clinical department one third the amount of service time (outpatient service, etc.) that the regular members of that department (of similar rank) devote to such service. He would owe the pharmacology department one third the amount of service time (student laboratory, etc.) that the regular full-time members of the department (of similar rank) devote to such service. The remaining one third of his service time would be devoted to his special services as a clinical pharmacologist, as described above. Experience has shown that departments with a strong, just organization, in which each full-time member of the department really does carry out his fair share of service, fit well into such a plan, for it is then possible to make a realistic fractionation. Thus, if each associate professor in the department of internal medicine ordinarily gives $\mathrm{X}$ hours of service, and each associate professor in the department of pharmacology gives $\mathrm{Y}$ hours of service, then an associate professor of pharmacology and internal medicine in the clinical pharmacology division would give one third $\mathrm{X}$ plus one third $\mathrm{Y}$ plus $\mathrm{Z}$ hours of service. $Z$ would represent service on the poison control center, assistance in teaching the human pharmacology seminar, special consultations in clinical pharmacology on the wards, etc. If the individual's talents and inclinations made it more reasonable for him to give two thirds $\mathrm{X}$ hours and no $\mathrm{Y}$ hours (or the reverse) this might be acceptable under certain circumstances.

\section{Additional considerations}

A. A major problem of the division of clinical pharmacology will lie in recruiting. Most of the young men now entering training have come to see success in the basic sciences as lying in the study of molecules and smaller particles. Success in clinical medicine is seen to lie in certification by the boards and training in an established subspecialty. Thus, a clinical pharmacology division should not expect to have a large number of trainees at the outset. It may, however, take advantage of the flexibility available to a new field. Examples of individuals who may profit from 1 year of training in the field as well as those who may profit from 2 and 3 years of training in the field have been presented. Examples of the various careers subsequently open to persons trained in clinical pharmacology have also been presented. These should be advertised judiciously but not blatantly.

B. It is to be hoped that divisions of clinical pharmacology will be able to reverse a trend that is now developing in certain medical schools. Pharmacologists as such are giving way to pure biochemists, etc. This is not to disparage the vitally important role of pure biochemists, etc., in medical schools but the waning influence of classical pharmacology is not a healthy development. Meanwhile, clinicians see an increasing need for medical students to receive proper background in pharmacology in preparation for the modern practice of medicine. In certain European countries, pharmacology has long been associated with the clinical years, and it would not be inconceivable for clinical groups to decide their students would require a new course in fundamentals of 
therapeutics, to be given in the clinical years, as a replacement for orthodox pharmacology, if pharmacology should become increasingly separated from clinical medicine. Such a development would probably not be a healthy one. Therefore, a division of clinical pharmacology may go far to stop the divorce proceedings which at present seem to be pending between two major areas in medicine. 